Bangladesh J. Plant Taxon. 26(1): 39-45, 2019 (June)

(C) 2019 Bangladesh Association of Plant Taxonomists

\title{
MOLECULAR CHARACTERIZATION AND NEW REPORTS OF TWO GREEN ALGAE FROM BANGLADESH
}

\author{
Md. Almujaddade Alfasane ${ }^{1}$, Md. Miraj Kobad Chowdhury ${ }^{2}$ \\ and MaLiha MehnaZ \\ Department of Botany, University of Dhaka, Dhaka-1000, Bangladesh
}

Keywords: Spirogyra maxima, Pithophora polymorpha, Molecular characterization, New reports, $18 \mathrm{~S}$ rDNA.

\begin{abstract}
This communication portrays the molecular characterization and confirms the new reports of two fresh water green algae namely, Pithophora polymorpha Wittrock and Spirogyra maxima (Hassall) Wittrock from Bangladesh. The samples of these algal species were cultured and partial $18 \mathrm{~S}$ rDNA was sequenced and analysed for their molecular identification. It was found that the primers reported here could sufficiently identify these algae as $P$. polymorpha and $S$. maxima. Furthermore, the Neighbourjoining (NJ) tree generated from 18s rDNA sequences suggested that Spirogyra maxima of Bangladesh is distantly related to the cluster of S. juergensii and S. platensis. Pithophora polymorpha along with $P$. roettleri, P. sano and Pithophora sp. seems to form a strongly supported monophyletic group. The alga AP1 clusters with Pithophora and the alga AS1 clusters with Spirogyra. This study is the first-time report of molecular identification of Bangladeshi algae and a landmark towards the future exploration of the algal biodiversity of Bangladesh.
\end{abstract}

\section{Introduction}

Algae are one of the important components and the most abundant primary producer of an ecosystem. Green algae represent a major biodiversity component of eukaryotic algae in continental water since they provide food by converting carbon dioxide to glucose and generate oxygen during photosynthesis (Barsanti and Gualtieri, 2014). Of them, filamentous green algae are of great economic value as they are the food sources of diverse aquatic animals and can be used to produce different products like paper and fibre despite often they are considered responsible for algal bloom (Nhat et al., 2018). Pithophora and Spirogyra are two common filamentous green algae found in tropical and temperate regions throughout the world including Bangladesh (Satpati and Pal, 2016). They are abundant in a wide range of freshwater habitats like small stagnant water bodies to running waters as they grow rapidly in eutrophic water and produce slimy green masses (Sarkar and Sekh, 2019). Pithophora is a genus of the order Cladophorales under the family of Pithophoraceae; and Spirogyra is a genus of filamentous green algae in the order Zygnematales under the family of Zygnemataceae (Moura-Júnior et al., 2016; Volkova et al., 2018). Pithophora resembles like a tangled mass of wool-like fibre and Spirogyra is easily recognized by the presence of spiral chloroplast. About 508 species of Spirogyra are now recognized whereas only 21 species of Pithophora have been reported (Boedeker et al., 2012; Stancheva et al., 2013). Identification of the Pithophora and Spirogyra species based only on morphological characteristics can be difficult because of their phenotypic plasticity and vast number of species. Thereby, molecular approaches are now in common practice to identify these algae (Thomson et al., 2018). Such approaches include PCR-RFLP, RAPD, AFLP, and partially or completely

\footnotetext{
${ }^{1}$ Corresponding author, Email: mujaddade@yahoo.com

${ }^{2}$ Department of Genetic Engineering and Biotechnology, University of Dhaka, Dhaka-1000, Bangladesh.
} 
sequencing of a conserved gene or genomic region (Manoylov, 2014). Among them, sequencing approaches is currently recognized as the best method with the advent of high-throughput technologies, and this tactic can sufficiently differentiate closely related species and even up to variety level in some cases (Lin et al., 2017). Genes proposed for such identification includes ISSR markers, rbcL gene, and 18S rDNA gene of algae (Haddad et al., 2014; Wongsawad and Peerapornpisal, 2014).

Bangladesh is enriched with freshwater ecosystem and about 2800 species of freshwater algae have been reported from Bangladesh. Hence, proper identification of these algae is very important to explore and conserve the algal biodiversity of Bangladesh. Although molecular identification of algae is now a common practice in different regions of the world, no report is available for the molecular identification of the algae of Bangladesh. This study aims to confirm the identification of two green algae from Bangladesh and their molecular characterization using partial sequencing of $18 \mathrm{~S}$ rDNA.

\section{Materials and Methods}

\section{Collection and morphological characterization}

Fresh filaments of Pithophora were collected from the Shoilo Propat fall, Bandarban $\left(22^{\circ} 10^{\prime} 48^{\prime \prime} \mathrm{N}, 092^{\circ} 13^{\prime} 48^{\prime \prime} \mathrm{E}\right)$, and fresh filaments of Spirogyra were collected from the Sangu river, Bandarban (22 $08^{\prime} 60^{\prime \prime} \mathrm{N}, 92^{\circ} 12^{\prime} 36^{\prime \prime}$ E), Bangladesh on 13 March 2017. These specimens were kept in source water and were transferred to the Limnology Laboratory of the Department of Botany, University of Dhaka within 24 hours of collection. As soon as the specimens arrived at the laboratory, they were examined under a light microscope as wet mounts and photomicrographs were taken using Nikon Eclipse E200. The cellular length, width, number and shape of chloroplasts as well as the number of granules in each filament were recorded for morphological characterization and taxonomic identification.

\section{Culture of algae}

Algae were cultured with the method as described before with some modifications (Sulfahri et al., 2017). Briefly, Bold's Basal Medium (BBM) was prepared before the collection of algae and was stored at $4^{\circ} \mathrm{C}$ after sterilization using $0.22 \mu \mathrm{m}$ filter. The $\mathrm{BBM}$ was warmed at $37^{\circ} \mathrm{C}$ before the culture of the algae. Individual filaments were transferred immediately to warmed BBM after microscopic observation for rescuing the algae and for purification of unialgal culture of the collected samples. The culture was incubated at $25^{\circ} \mathrm{C}$ for five to seven days in an orbital shaker at $125 \mathrm{rpm}$ under $150 \mu \mathrm{E} / \mathrm{m}^{2} / \mathrm{s}$ intensity of light with $12: 12$ light/dark cycle, growth of filaments was observed time to time and the filaments were sub-cultured following the technique mentioned above to purify the algae.

\section{DNA isolation and PCR}

For molecular characterization and identification of species, partial sequences of $18 \mathrm{~S}$ rDNA was amplified using the primers: Forward 5'-AGGGC AAGTC TGGTG CCAGCAG-3' and Reverse 5'-GTTGA GTCAA ATTAA GCCGC-3'. Genomic DNA was extracted using modified phenol-chloroform-isoamyl alcohol method (Tabrejee et al., 2018). Briefly, individual algal culture was rinsed with distilled water followed by with $70 \%$ ethanol. Then the filaments were airdried to remove excess ethanol. The filaments were grinded to fine powder using liquid nitrogen. About $100 \mathrm{mg}$ homogenized tissue was mixed with $500 \mu \mathrm{l}$ of CTAB extraction buffer ( $2 \%$ cetyl trimethylammonium bromide, $1 \%$ polyvinyl pyrrolidone, $100 \mathrm{mM}$ Tris- $\mathrm{HCl}, 1.4 \mathrm{M} \mathrm{NaCl}, 20 \mathrm{mM}$ EDTA) and was vortexed thoroughly. The homogenate was transferred to a $60^{\circ} \mathrm{C}$ water bath for 30 minutes. Then the homogenate was centrifuged for 5 minutes at $14,000 \mathrm{x} g$ and the supernatant 
was collected for DNA extraction. The DNA was estimated using NanoDrop ${ }^{\mathrm{TM}}$ and was used for PCR. The PCR condition includes an initial step of 5 min at $95{ }^{\circ} \mathrm{C}$, then 40 cycles of 30 seconds at $95^{\circ} \mathrm{C}, 30$ seconds at $57^{\circ} \mathrm{C}$, and $1 \mathrm{~min}$ at $72^{\circ} \mathrm{C}$, followed by $10 \mathrm{~min}$ at $72^{\circ} \mathrm{C}$. The PCR products were visualized on $1 \%$ agarose gel under UV-transilluminator and photomicrograph was taken.

Sequencing and phylogenetic analysis

To design the primers, multiples sequences of $18 \mathrm{~S}$ rDNA gene (Table 1) were aligned using Clustal Omega. From the alignment, two conserved regions spacing 600-800 bp were selected (Fig. 1). Sequences from these regions were used for primer design. Next, these primers were verified using Primer-BLAST tool. The PCR products were purified using PureLink ${ }^{\mathrm{TM}}$ PCR purification kit and sequenced at the Macrogen, South Korea by Sanger sequencing. All the raw sequences were processed using FinchTV and aligned using CLC workbench. These sequences were aligned using Basic Local Alignment Search Tool (BLAST) with the 18S rDNA sequences database of the National Center for Biotechnology Information (NCBI) for molecular identification and were submitted to GenBank with referred accession numbers (MH894274MH894275). Nucleotide compositions of the processed sequences were analysed using Mega v5.05. Neighbour-joining (NJ) tree based on K2P distances was created to illustrate molecular phylogeny using Mega v5.05. For this, Vampyrella lateritia was used as an outgroup.

\begin{tabular}{|c|c|c|}
\hline Sequences & Forward Primer & Reverse Primer \\
\hline 1892869 & ggcaagtctggtgccagcá & cacrggaaactta \\
\hline & gagggcaagtctggtgccagcagccgcggtaattccạ & acacgggaaaactta \\
\hline Aa_FR873097 & gcagccgcggtaattccạ & cacgggaaaactta \\
\hline 646 & gagggcaagtctggtgccagcagccgcggtaattccạ & cacgggaaaactta \\
\hline & gagggcaagtctggtgccagcagccgcggtaattccạ & acacgggaaactta \\
\hline 241 & gagggcaagtctggtgccagcagecgcggtaattccac & caacacgggaaaactta \\
\hline 30 & cagccgcggtaattccac & acacgg \\
\hline 75 & cagccgcggtaattccạ & aatcte $\bar{c}$ \\
\hline 23 & gcagccgcggtaattccạ & cacggggaaantta \\
\hline $\bar{J} Q 290272$ & gagggcaagtctggtgccagcagccgcggtaattccac & acgcggggaatctta \\
\hline & gagggcaagtctggtgccagcagccgcggtaattccạ & cgtgcggcttaatttgactcaacgcggggaatctta \\
\hline 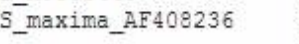 & gcagccgeggtaattccạ & 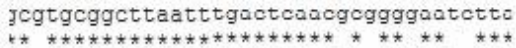 \\
\hline
\end{tabular}

Fig. 1. Multiple sequence alignment using different $18 \mathrm{~S}$ rDNA sequences. Two conserved regions spacing 600-800 bp were selected for the designing of forward and reverse primer as indicated by arrow.

\section{Results and Discussion}

Here, two different algae from Bangladesh were studied in classical morphology method as well as molecular method (Barsanti and Gualtieri, 2014). These algae were collected and were successfully cultured using BBM. While studied under the light microscope, it was observed that the collected algal filaments were either of Pithophora genus or of Spirogyra genus. The filaments of Pithophora were green to dark brown in color, freely but sparsely branched, and containing intercalary and terminal akinetes (Fig. 2A). Cells of Pithophora were slender and cylindrical with $1100-1450 \mu \mathrm{m}$ length and 50-120 $\mu \mathrm{m}$ width comprising thin cell wall without layers. Each cell contained one reticulated chloroplast with numerous pyrinoids (Fig. 2B). Terminal cells are conical and rounded. These data were consistent with previous reports (Manoylov, 2014; MouraJúnior et al., 2016). Filaments of Spirogyra were light green to green and unbranched. Vegetative cells of Spirogyra were $70-100 \mu \mathrm{m}$ wide and $120-230 \mu \mathrm{m}$ long. Cell wall plane was transverse, and each cell contained 5-7 spiral chloroplasts with numerous pyrinoids (Fig. 2C,D). Brown, multilayered, and reticulated mesospores were present in the filament. Often, lenticular zygospores were present in the filaments of Spirogyra. Such observations were similar to previous findings (Manoylov, 2014; Volkova et al., 2018). However, confirming the species of these algae based on 
such morphological observations was difficult. Hence, molecular characterization of these algae was applied based on partial $18 \mathrm{~S}$ rDNA typing to confirm the genus and to identify the species.

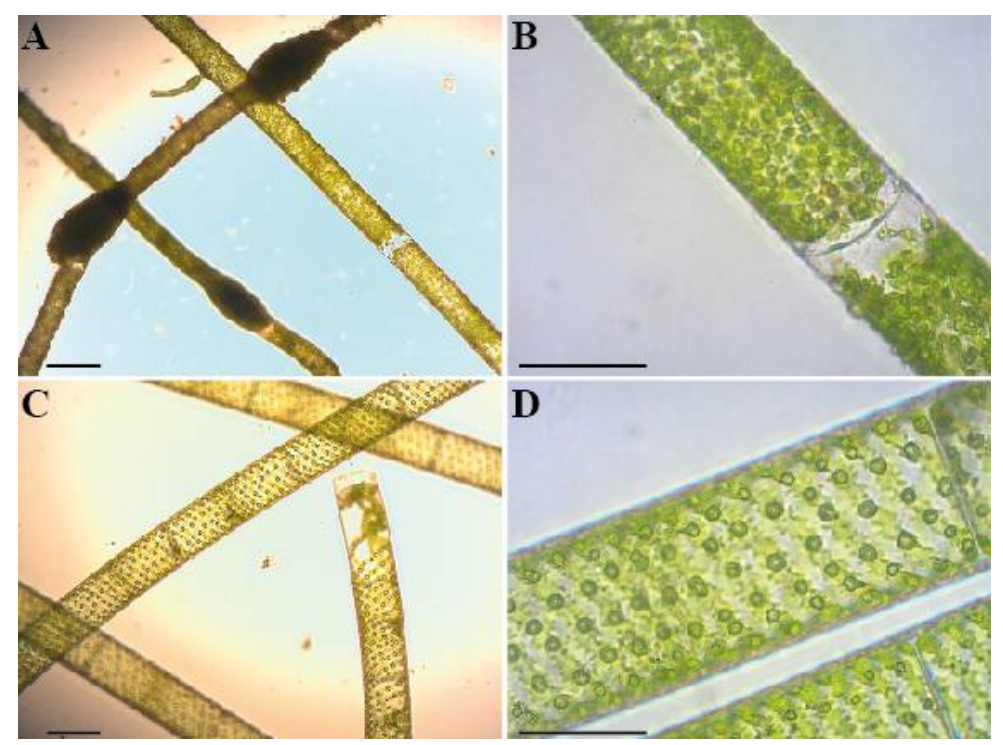

Fig. 2. Representative photomicrograph of Pithophora and Spirogyra filaments. Akinetes (A) and reticulated (B) chloroplasts of Pithophora filaments, distinct green filaments (C) and spiral chloroplasts (D) of Spirogyra. Bar $=50 \mu \mathrm{m}$.

For such molecular characterization, genomic DNA was extracted and purified from these samples. The quality and quantity were analysed using NanoDrop ${ }^{\mathrm{TM}}$ and the A260/A280 ratio was around $1.80-1.82$, indicating the quality of the isolated DNA was quite good. The quantity of isolated DNA was 260-420 ng/ $\mu$ l. Moreover, when electrophoresed through an agarose gel, little fragmentation or smear was observed indicating that majority of the DNA was almost intact or partially fragmented (Fig. 3A). When the $18 \mathrm{~S}$ rDNA region was partially amplified from these DNA samples by PCR, a distinct band near 650 bp was observed in both cases (Fig. 3B). Thus, it can be concluded that the isolated DNA was in good quality and the partial $18 \mathrm{~S}$ rDNA region can be amplified by the designed primers.
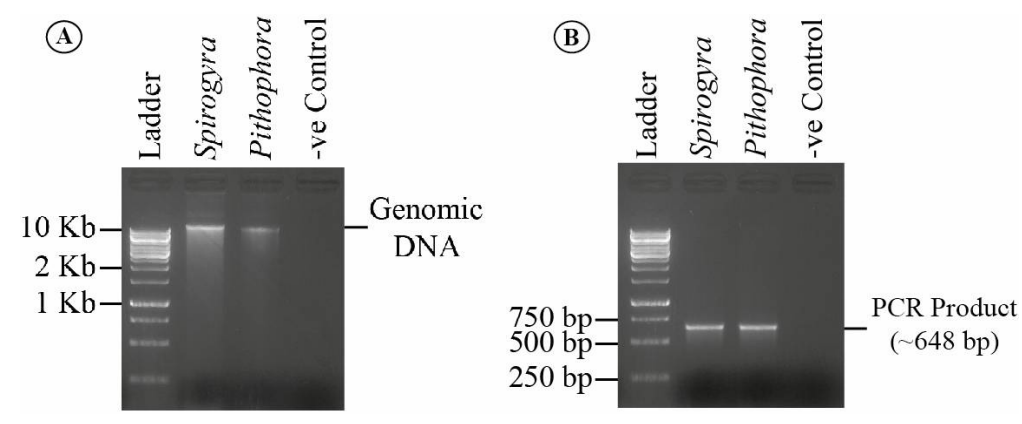

Fig. 3. Agarose gel electrophoresis of isolated genomic DNA (A) and partially amplified 18S rDNA PCR products (B) of Spirogyra and Pithophora. A clear band of about 648 bp was observed after PCR amplification. 
The PCR product was sequenced and aligned with the available 18S rDNA sequences at NCBI using BLAST for molecular identification. The sequences of Pithophora matched mostly with the available Pithophora polymorpha $18 \mathrm{~S}$ rDNA sequences (Identity $=100 \%$; E-value $=0.0$ ). And, the sequences of Spirogyra matched mostly with the available Spirogyra maxima 18S rDNA sequences (Identity $=91 \%$; E-value $=8 \mathrm{e}^{-127}$ ). Thereby, it can be concluded that isolated algae were Pithophora polymorpha and Spirogyra maxima, respectively. The Neighbour-joining (NJ) tree constructed using the partial sequences of 18s rDNA (Table 1) showed that the sampled taxa of Spirogyra and Pithophora clustered separately (Fig. 4). In the Neighbour-joining (NJ) tree, Spirogyra maxima of Bangladesh seems to form a cluster with alga AS1 clusters but distantly related to the cluster of $S$. juergensii and S. platensis, previously published species of Spirogyra (Wongsawad and Peerapornpisal, 2014). Pithophora polymorpha along with P. roettleri, P. sano, Pithophora sp. and alga AP1 clusters seems to form a strongly supported larger cluster.

Table 1. Partial 18S rDNA sequences and their accession numbers used for multiple sequence alignment for primer designing.

\begin{tabular}{ll}
\hline Sequence & Accession number \\
\hline Spirogyra juergensii & JQ290272 \\
Spirogyra maxima & AF408236 \\
Spirogyra platensis & JQ290275 \\
Spirogyra grevilleana & U18523 \\
Spirogyra sp. & AJ853449 \\
Pithophora polymorpha & FR873097 \\
Pithophora roettleri & FR719930 \\
Pithophora sano & AB066646 \\
Pithophora sp. & KM892869 \\
Pithophora sp. & KU727241 \\
Pithophora $s p$. & KU727242 \\
Pithophora sp. & KU727240 \\
\hline
\end{tabular}

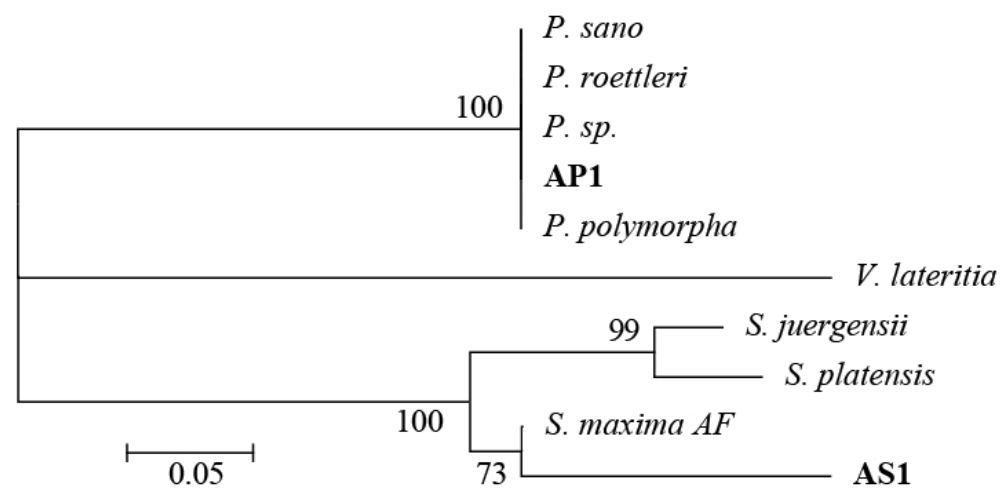

Fig. 4. Phylogenetic tree (NJ) constructed from partial 18s rDNA sequences of Pithophora (AP1) and Spirogyra (AS1). Scale bar represents genetic distance and Vampyrella lateritia was used as an outgroup organism. The alga AP1 clusters with Pithophora and the alga AS1 clusters with Spirogyra. 
This study accounts for the first report on molecular characterization of algae found in Bangladesh. Both the Pithophora polymorpha and the Spirogyra maxima were successfully identified by analysing partial $18 \mathrm{~S}$ rDNA sequences and these two algae are also new reports for Bangladesh. Development of a complete dataset on 18S rDNA sequences of all the algae found in Bangladesh is required for future identification of algal species and for the conservation of algal biodiversity of Bangladesh.

\section{Acknowledgments}

The authors wish to thank Ministry of Science and Technology, Government of the People's Republic of Bangladesh for funding this research.

\section{Conflicts of Interest}

The authors declare no conflicts of interest.

\section{References}

Barsanti, L. and Gualtieri, P. 2014. Algae : anatomy, biochemistry, and biotechnology, CRC Press. Second Edition. pp. 1-361.

Boedeker, C., O'Kelly, C.J., Star, W. and Leliaert, F. 2012. Molecular Phylogeny and Taxonomy of the Aegagropila Clade (Cladophorales, Ulvophyceae), Including the Description of Aegagropilopsis Gen. Nov. And Pseudocladophora. J. Phycol. 48(3): 808-825.

Haddad, R., Alemzadeh, E., Ahmadi, A.R., Hosseini, R. and Moezzi, M. 2014. Identification of Chlorophyceae based on 18S rDNA sequences from Persian Gulf. Iran J. Microbiol. 6(6): 437-442.

Lin, G.M., Lai, Y.H., Audira, G. and Hsiao, C. D. 2017. A Simple Method to Decode the Complete 18-5.828S rRNA Repeated Units of Green Algae by Genome Skimming. Int. J. Mol. Sci. 18(11): 23-41.

Manoylov, K. M. 2014. Taxonomic identification of algae (morphological and molecular): species concepts, methodologies, and their implications for ecological bioassessment. J. Phycol. 50(3): 409-424.

Moura-Júnior, E.G.D., Valentin-de-Souza, R.L. and Milhomens, L.B.S. 2016. New record of Pithophora roettleri (Roth) Wittrock, 1877 (Chlorophyta: Pithophoraceae) for the São Francisco River basin and its potential geographic distribution in Brazil. Check List. 12(3): 18-99.

Nhat, V.H.P., Ngo, H.H., Guo, W.S., Chang, S.W., Nguyen, D.D. and Nguyen, P.D. 2018. Can algae-based technologies be an affordable green process for biofuel production and wastewater remediation? Bioresour. Technol. 256: 491-501.

Sarkar, N.S. and Sekh, S. 2019. Dynamics of Algae in Sundarbans and their Implications as Climate Change Proxies. The Sundarbans: A Disaster-Prone Eco-Region: Increasing Livelihood Security. H. S. Sen. Cham, Springer International Publishing. pp. 133-159.

Satpati, G. G. and Pal, R. 2016. New and rare records of filamentous green algae from Indian Sundarbans Biosphere Reserve. J. Algal Biomass Utln. 7(2): 159-175.

Stancheva, R., Hall, J.D., McCourt, R.M. and Sheath, R.G. 2013. Identity and phylogenetic placement of Spirogyra species (Zygnematophyceae, Charophyta) from California streams and elsewhere. J. Phycol. 49(3): 588-607.

Sulfahri, Amin, M., Sumitro, S.B. and Saptasari, M. 2017. Comparison of biomass production from algae Spirogyra hyalina and Spirogyra peipingensis. Biofuels 8(3): 359-366.

Tabrejee, S., Chowdhury, M. M. K., Bari, L. and Yeasmin, S. 2018. Isolation and Characterization of Enterococci from Yoghurts of Bangladesh. Mod. Care J. 15(3): e74460.

Thomson, S.A., Pyle, R.L., Ahyong, S.T., Alonso-Zarazaga, M., Ammirati, J. and Araya, J.F. 2018. Taxonomy based on science is necessary for global conservation. PLoS Biol. 16(3): e2005075. 
Volkova, E.A., Bondarenko, N.A. and Timoshkin, O.A.Y. 2018. Morphotaxonomy, distribution and abundance of Spirogyra (Zygnematophyceae, Charophyta) in Lake Baikal, East Siberia. Phycologia 57(3): 298-308.

Wongsawad, P. and Peerapornpisal, Y. 2014. Molecular identification and phylogenetic relationship of green algae, Spirogyra ellipsospora (Chlorophyta) using ISSR and rbcL markers. Saudi J. Biol. Sci. 21(5): 505-510.

(Manuscript received on 2 February, 2019; revised on 28 April, 2019) 